\title{
Selective excitations of intrinsic localized modes of atomic scales in carbon nanotubes
}

\section{$\operatorname{AUTHOR}(\mathrm{S}):$}

Kinoshita, Y; Yamayose, Y; Doi, Y; Nakatani, A; Kitamura, $T$

\section{CITATION:}

Kinoshita, Y ... [et al]. Selective excitations of intrinsic localized modes of atomic scales in carbon nanotubes. PHYSICAL REVIEW B 2008, 77(2): 024307.

\section{ISSUE DATE:}

2008-01

URL:

http://hdl.handle.net/2433/84623

RIGHT:

(C) 2008 The American Physical Society 
PHYSICAL REVIEW B 77, 024307 (2008)

\title{
Selective excitations of intrinsic localized modes of atomic scales in carbon nanotubes
}

\author{
Y. Kinoshita, ${ }^{1}$ Y. Yamayose, ${ }^{2}$ Y. Doi ${ }^{3}$ A. Nakatani,${ }^{3}$ and T. Kitamura ${ }^{1}$ \\ ${ }^{1}$ Department of Mechanical Engineering and Science, Kyoto University, Yoshida-hommachi, Sakyo-ku, Kyoto-shi, \\ Kyoto 606-8501, Japan \\ ${ }^{2}$ Research and Development Center, Toshiba Corporation, 1, Komukai Toshiba-cho, Saiwai-ku, Kawasaki-shi, \\ Kanagawa 212-8582, Japan \\ ${ }^{3}$ Department of Adaptive Machine Systems, Osaka University, Suita, Osaka 565-0871, Japan
}

(Received 8 August 2007; revised manuscript received 18 October 2007; published 16 January 2008)

\begin{abstract}
In order to examine the excitation of intrinsic localized modes (ILMs) in a three-dimensional material, we conducted molecular dynamics numerical simulations on $(12,0),(10,0)$, and $(8,0)$ zigzag carbon nanotubes (CNTs) and (7,7), (6,6), and $(5,5)$ armchair CNTs based on the Brenner potential. While energy localization is observed in several regions in the zigzag CNTs, it is not seen in the armchair CNTs. In the former, fairly constant modes, where two neighboring atoms vibrate in the opposite direction along the axial direction, are found in the energy-localized region, and their frequencies exceed the upper bound of the phonon band. In the armchair CNTs, atomic vibrations in the circumferential direction within high-energy regions cannot last a long time. These results indicate that the ILM is excited in the three zigzag CNTs but not in the three armchair ones. This is because the bond along the tube-axial direction has stronger nonlinearity under vibrations than that in the circumferential one, and the bond direction depends on the structure of the CNT.
\end{abstract}

DOI: 10.1103/PhysRevB.77.024307

PACS number(s): 05.45.Yv, 63.20.Ry, 63.22.-m

\section{INTRODUCTION}

Since the finding of the intrinsic localized mode (ILM) or discrete breather by Takeno and co-workers in $1988,{ }^{1,2}$ it has been studied extensively by many researchers, both theoretically and experimentally. ${ }^{3-16}$ The ILM is a time-periodic and spatially localized vibration mode which appears in lattice systems consisting of discrete elements with nonlinear interaction. Both the nonlinearity and the discreteness excite the ILM because (i) waves with a frequency exceeding the upper bound of the phonon band $\omega_{\max }$ cannot spread in a system of discrete elements, (ii) the nonlinearity in the interaction between the elements tends to wake waves with relatively large amplitude, and (iii) some nonlinear waves can have a frequency higher than $\omega_{\max }$.

Existence of the stationary ILM has been proved in a wide range of lattice systems. ${ }^{4}$ Recently, the ILM has been observed experimentally in various physical contexts, such as Josephson junction arrays, ${ }^{5,6}$ optical lattices, ${ }^{7}$ and micromechanical cantilever arrays. ${ }^{8,9}$ Adding to these interesting results, the ILM in real crystals is worth studying. The ILM may appear in atomic systems with a crystal structure because of the discreteness of their structures and the nonlinearity in the interaction between atoms. While most studies on the ILM have dealt with one-dimensional lattice systems, ${ }^{10-13}$ some studies of the ILM have been carried out for crystal structures under imaginary potentials. Cuevas $e t$ al. numerically analyzed the effects of a vacancy on the ILM in a crystal using an anharmonic interatomic potential of model material. ${ }^{14}$ Marín et al. studied the excitation of the moving ILM in L-J crystals using simple molecular dynamics simulations. ${ }^{15}$ They reported that the moving ILM which is initially excited survives in 7000 time units (1344 lattice cites) or more. Moreover, Russel and Eilbeck has claimed experimental evidence which implies the excitation of the ILM in a crystal. ${ }^{16}$ However, there are some gaps between the theoretical or numerical results and this experiment. Thus, more detailed analysis is required for the verification of the ILM in crystals.

The present authors analyzed two-dimensional vibrations of a graphene sheet by molecular dynamics simulation using a realistic potential (Brenner potential ${ }^{17}$ ) and validated the excitation of the ILM on the basis of the life and the frequency of localized wave. ${ }^{18}$ This result suggests the excitation of the ILM in three-dimensional materials.

The carbon nanotube (CNT), which has been attracting attention because of its superior mechanical strength, high chemical and thermal resistance, etc., ${ }^{19-21}$ is made by rolling up a graphene sheet. Therefore, the CNT may have the ILM as a three-dimensional material. Different CNT structures are obtained by changing the direction and length of rolling of a graphene sheet (see next section) and may influence the excitation of the ILM. Savin and Kivshar studied the excitation of the ILM in $(m, 0)$ and $(m, m)$ CNTs. ${ }^{22}$ They showed that three types of ILMs can exist in the $(m, 0)$ CNT, namely,

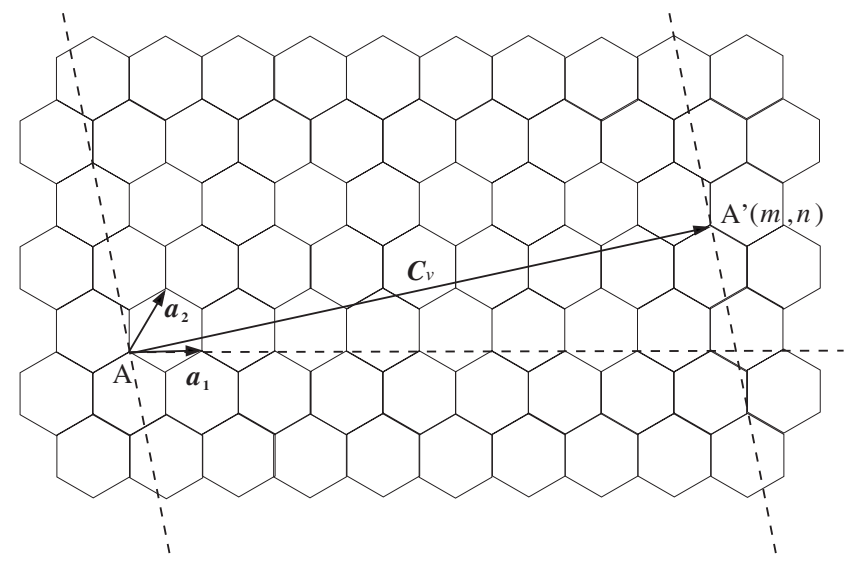

FIG. 1. Schematic illustration explaining the chiral vector of a carbon nanotube. 
TABLE I. Simulation models of zigzag and armchair CNTs.

\begin{tabular}{cccc}
\hline \hline Chiral index & $\begin{array}{c}\text { Diameter } \\
(\mathrm{nm})\end{array}$ & $\begin{array}{c}\text { Length } \\
(\mathrm{nm})\end{array}$ & No. of atoms \\
\hline$(12,0)$ & 0.962 & 3.055 & 336 \\
$(10,0)$ & 0.803 & 3.059 & 280 \\
$(8,0)$ & 0.644 & 3.066 & 224 \\
$(7,7)$ & 0.972 & 3.023 & 336 \\
$(6,6)$ & 0.834 & 3.026 & 288 \\
$(5,5)$ & 0.696 & 3.031 & 240 \\
\hline \hline
\end{tabular}

longitudinal, radial, and twisting ILMs, while the $(m, m)$ CNT allows only the excitation of the radial ILM. However, they modeled the CNTs by one-dimensional diatomic chains and adopted model potentials, which indicates that further studies are required to elucidate the ILM in CNTs as a real three-dimensional material.

In the present study, we have examined the excitation of the ILM in $(12,0),(10,0)$, and (8.0) zigzag CNTs and $(7,7)$, $(6,6)$, and $(5,5)$ armchair CNTs using molecular dynamics simulations and discuss the effects of the atomic structure.

\section{SIMULATION PROCEDURE}

Figure 1 shows the unit vectors $\boldsymbol{a}_{1}$ and $\boldsymbol{a}_{2}$ of the hexagonal honeycomb lattice of graphene. The circumference of any CNT is uniquely represented by the chiral vector,

$$
\boldsymbol{C}_{v}=m \boldsymbol{a}_{1}+n \boldsymbol{a}_{2} \equiv(m, n),
$$

which connects two crystallographically equivalent sites on a graphene. The zigzag $(n=0)$ and armchair $(m=n)$ CNTs have a characteristic structure showing no helicity. In the present work, we focus on three zigzag and three armchair CNTs, for which the calculated cell sizes are shown in Table I. The periodic boundary condition is applied in the axial direction. We have confirmed that if the length of CNTs is longer than $3 \mathrm{~nm}$, the number of ILMs excited per unit length does not change and the lifetime of ILMs is not affected by the length, namely, there is no finite size effect in this study.

The Hamiltonian of the system is given by

$$
H=\sum_{i}^{N} \sum_{\alpha} \frac{\left(p_{i}^{\alpha}\right)^{2}}{2 M}+\frac{1}{2} \sum_{i}^{N} \sum_{j \neq i}^{N} \sum_{k \neq i, j}^{N} \Phi_{i j k}\left(r_{i j}, r_{i k}, \theta_{i j k}\right),
$$

where $N$ is the number of carbon atoms in a calculation cell, $i, j$, and $k$ are the indices of the carbon atoms, $\alpha$ is the
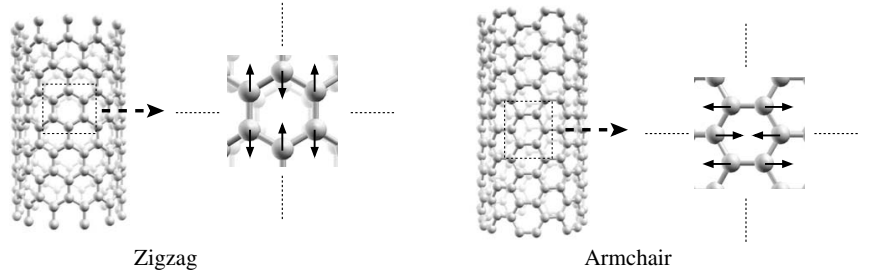

FIG. 2. Schematic illustration explaining initial displacements.

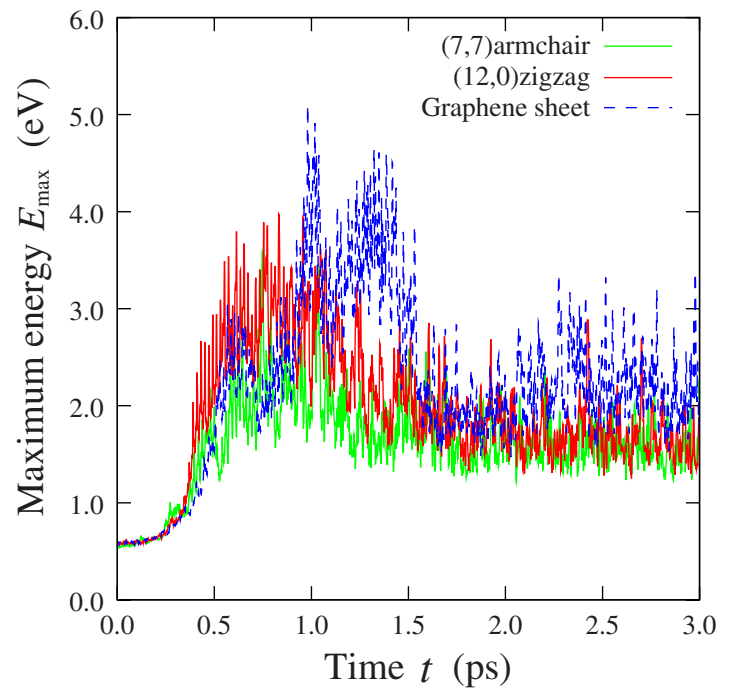

FIG. 3. (Color online) Change in the maximum energy of the $(7,7) \mathrm{CNT},(12,0) \mathrm{CNT}$, and graphene sheet.

coordinate $x, y$, or $z, p_{i}^{\alpha}$ is the momentum of the $i$ th atom in the $\alpha$ direction, $M$ is the mass of the carbon atom, $\Phi_{i j k}$ is the interatomic potential, $r_{i j}$ is the distance between atoms $i$ and $j$, and $\theta_{i j k}$ is the angle between bonds $i-j$ and $i-k$. An empirical bond order potential proposed by Brenner, ${ }^{17}$ which can describe the mechanical properties of carbons, ${ }^{23}$ is adopted as the functional $\Phi_{i j k}$ in the simulation. Brenner proposed two sets of parameters. One can precisely reproduce the bond length between atoms, though it has a relatively large error in the calculation of atomic force. The other gives reasonable bond lengths and forces with errors of several percent. In the present study, the latter is adopted because it is suitable for the dynamic simulation.

We investigate the excitation of the ILM from specific initial conditions. It is well known that the ILM is excited from the modulational instability of the phonon mode which has the maximum angular frequency (zone boundary mode). ${ }^{24}$ We apply displacement with the same amplitude

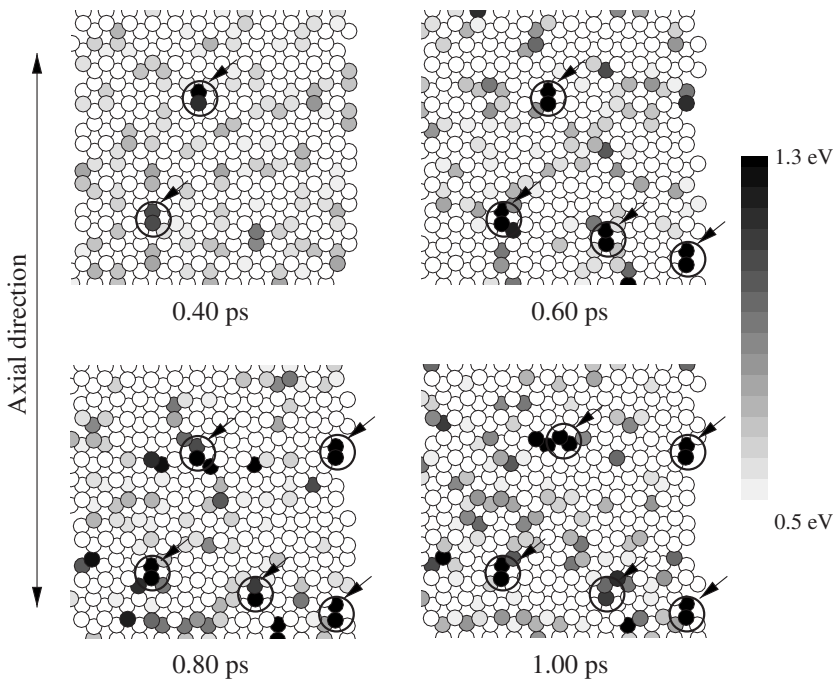

FIG. 4. Change in the distribution of total energy in the $(12,0)$ CNT. 


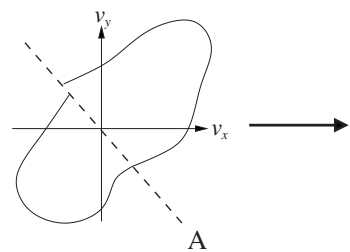

(a)

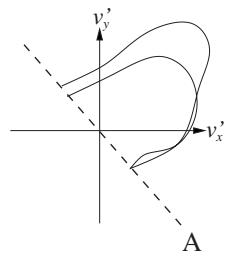

(b)
FIG. 5. Schematic illustration of the velocity space of atom $i$ for explaining the extraction procedure for the vibration mode of atom $i, \boldsymbol{V}_{i}$.

and momentum with a minute disturbance to all atoms. As shown in Fig. 2, an initial displacement of $0.01 \mathrm{~nm}$ is applied to the axial direction in the zigzag CNTs and to the circumferential direction in the armchair CNTs, respectively, because the nonlinearity in the potential promotes the excitation of ILM. Initial random momentum with a normal distribution is applied to each atom. Here, the initial temperature is $10 \mathrm{~K}$ because thermal vibrations under the higher temperatures make it difficult to study localized vibrations. The Verlet algorithm ${ }^{25}$ is used for the numerical integration under the time step of $0.01 \mathrm{fs}$, the reason of which is that the larger time steps cause non-negligible truncation error leading to the impossibility of extracting the exact trajectory of atoms, namely, the impossibility of analyzing the exact vibration of atoms. The molecular dynamics simulation is conducted under the microcanonical condition.

\section{RESULTS AND DISCUSSION}

The Brenner potential allows us to calculate the potential energy of an arbitrary atom $i, E_{i}^{\text {pot}}$;

$$
E_{i}^{\mathrm{pot}}=\frac{1}{2} \sum_{j \neq i}^{N} \sum_{k \neq i, j}^{N} \Phi_{i j k} .
$$

Since the kinetic energy of an atom $i, E_{i}^{\mathrm{kin}}$, can be calculated by the momentum, we obtain the total energy of atom $i$ as $E_{i}=E_{i}^{\mathrm{pot}}+E_{i}^{\mathrm{kin}}$.

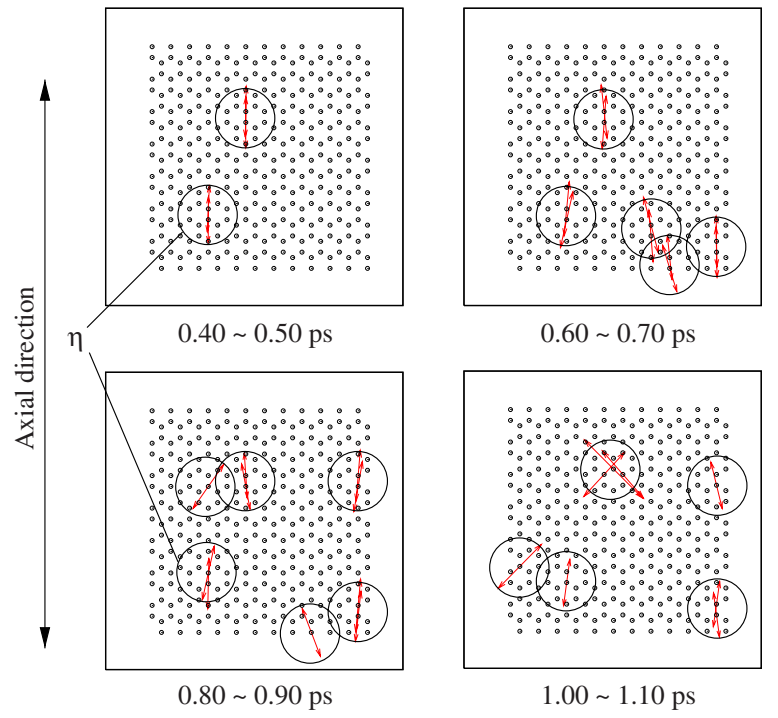

FIG. 6. (Color online) Vibration mode of atoms in the $(12,0)$ zigzag CNT. $\boldsymbol{V}_{i}$ with large norms (larger than $75 \%$ of its maximum) are indicated by arrows in the figure.

Figure 3 shows the largest energy of an atom, $E_{\max }$ $=\max \left(E_{i}\right)$, in the $(7,7)$ armchair and $(12,0)$ zigzag CNTs. $E_{\text {max }}$ of the graphene sheet ${ }^{18}$ is also shown for comparison. For both armchair and zigzag CNTs, after the magnitude of $E_{\max }$ increases significantly, it remains high from 0.50 to $1.20 \mathrm{ps}$, though the increase in the CNTs is smaller than that in the graphene sheet.

Figure 4 shows the change in the distribution of energy in the $(12,0)$ CNT. The atoms indicated by the arrows possess higher energy and maintain the state for more than 300 fs. In other words, there appear to be areas of energy localization on the atomic scale for a fairly long lifetime. The energy is localized in the region of two atoms, which corresponds to a characteristic feature of the ILM. None of these atoms undergo migration, which suggests excitation of the stationary ILM.

Figure 5 shows a procedure for extracting the vibration mode of each atom. Since the velocity represents the direction of movement, the dominant moving direction of an atom

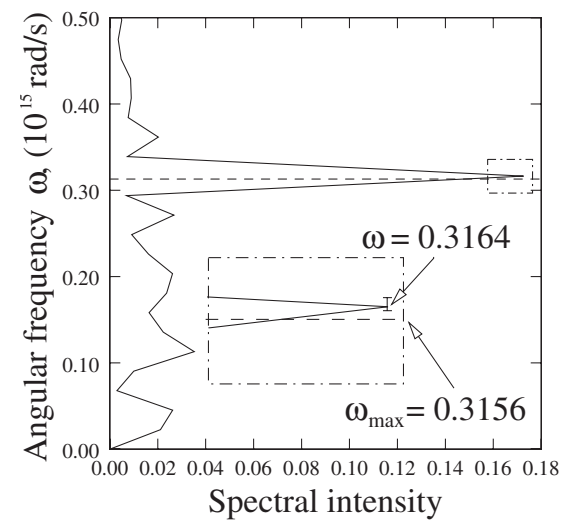

(a) $(12,0)$

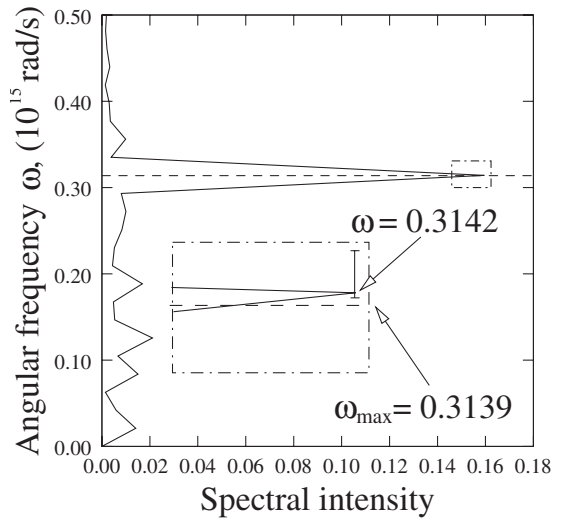

(b) $(10,0)$

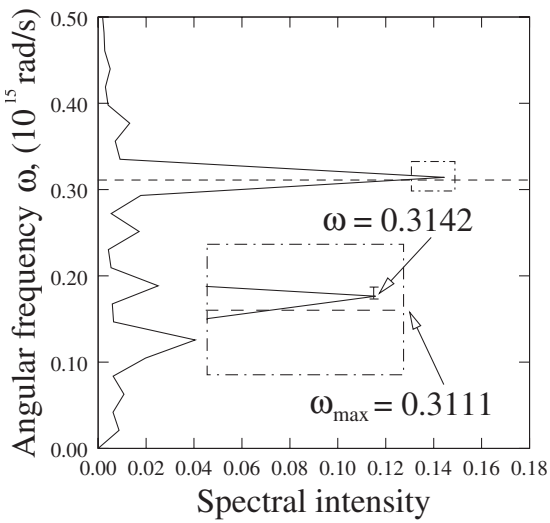

(c) $(8,0)$

FIG. 7. Power spectrum distribution in the $(12,0),(10,0)$, and $(8,0)$ zigzag CNTs. 


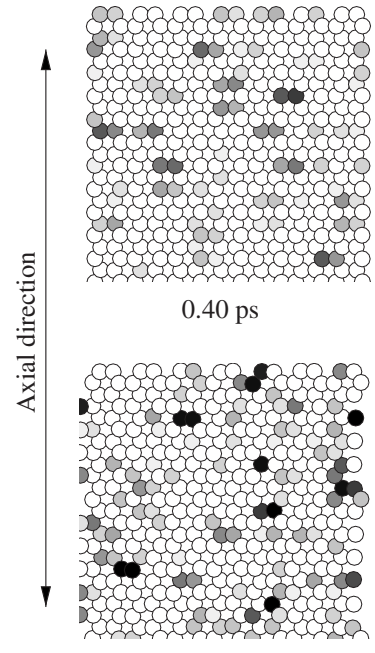

$0.80 \mathrm{ps}$

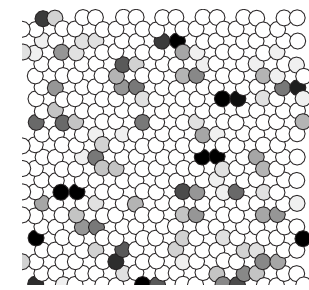

$0.60 \mathrm{ps}$

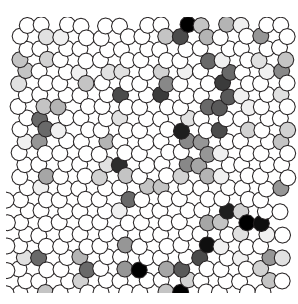

$1.00 \mathrm{ps}$

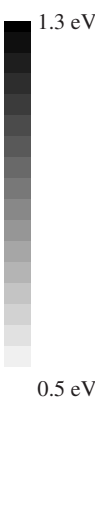

FIG. 8. Change in the distribution of total energy in the $(7,7)$ CNT.

during a time interval can be extracted by averaging the velocity of the atom over the time interval. Note that if we normally do it, the average velocity of an atom vibrating around its equilibrium point becomes almost zero, resulting in the impossibility of extracting the dominant vibration mode of the atom. To resolve this issue, the trajectory of the velocity vector $\boldsymbol{v}_{i}(t)$ of atom $i$ from $t$ to $t+\delta t$ [Fig. 5(a)] is folded back along a straight line A passing through the origin [Fig. 5(b)], where the folded velocity vector is denoted by $\boldsymbol{v}_{i}^{\prime}(t)$. Here, $\delta t$ is the period of vibration. Then, a vector $\boldsymbol{V}_{i}$ is defined as the time average of $\boldsymbol{v}_{i}^{\prime}(t)$,

$$
\boldsymbol{V}_{i}=\frac{\int_{t}^{t+\delta t} \boldsymbol{v}_{i}^{\prime}(t) d t}{\delta t} .
$$

The straight line $\mathrm{A}$ is determined so as to maximize the norm of $\boldsymbol{V}_{i}$, which represents the vibration mode of atom $i$ during the time interval, $\delta t$. The norm becomes large as the atom vibrates faster in a particular direction. Figure 6 shows $\boldsymbol{V}_{i}$ at every $0.10 \mathrm{ps}$, of which the norm is larger than $75 \%$ of its

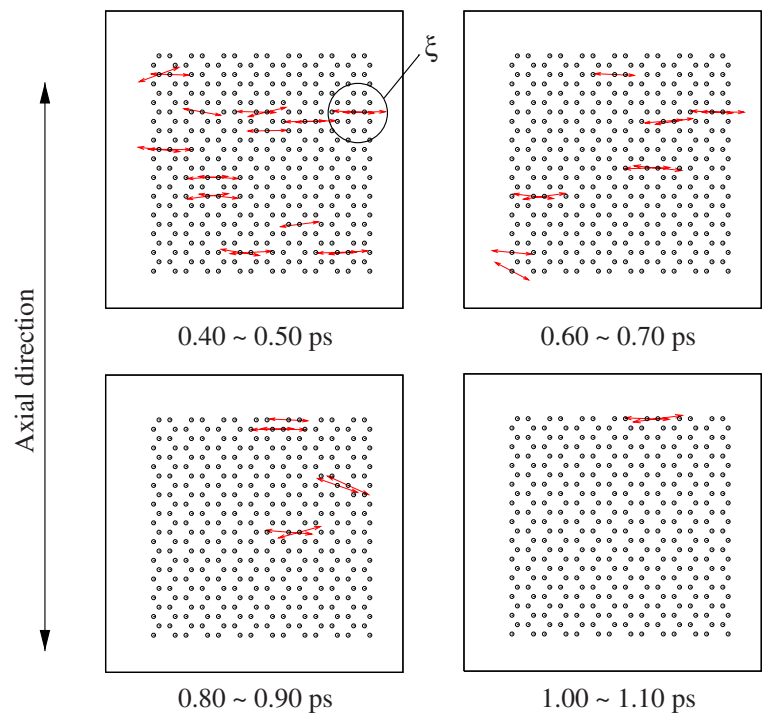

FIG. 9. (Color online) Vibration mode of atoms in the $(7,7)$ armchair CNT. $V_{i}$ with large norms (larger than $75 \%$ of its maximum) are indicated by arrows in the figure.

maximum in the $(12,0) \mathrm{CNT}$. This reveals that fairly constant vibration modes, in which the two neighboring atoms vibrate in the opposite direction along the axial direction, are formed at the localized areas. These modes correspond well to the areas of excited waves indicated in Fig. 4. A vibration with an opposite phase (optical mode) is one of the characteristic features of the ILM.

The phonon dispersion is obtaind by diagonalizing the dynamical matrix $D$,

$$
D(\boldsymbol{k})=\sum_{l^{\prime}} \frac{1}{\sqrt{M_{i} M_{j}}} K_{l i \alpha}^{l^{\prime} j \beta} \exp \left[i \boldsymbol{k} \cdot\left(\boldsymbol{R}_{l^{\prime} j}-\boldsymbol{R}_{l i}\right)\right],
$$

where subscripts $l$ and $l^{\prime}$ are the numbers of unit cells, $i$ and $j$ are the indices of atoms in the unit cell, $\alpha$ and $\beta$ are the $x$, $y$, or $z$ coordinates, $\boldsymbol{R}_{l i}$ represents the space coordinates of the $i$ th atom in the $l$ th cell, and $\boldsymbol{k}$ is the wave number vector. The force tensor $K_{l i \alpha}^{l^{\prime} j \beta}$ is defined as the second derivative of the potential energy $P$, with respect to the atomic coordinates,

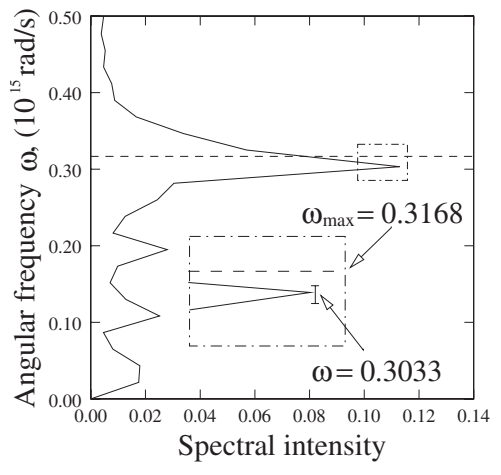

(a) $(7,7)$

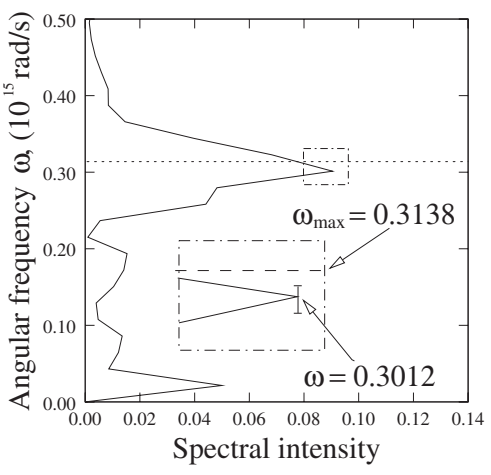

(b) $(6,6)$

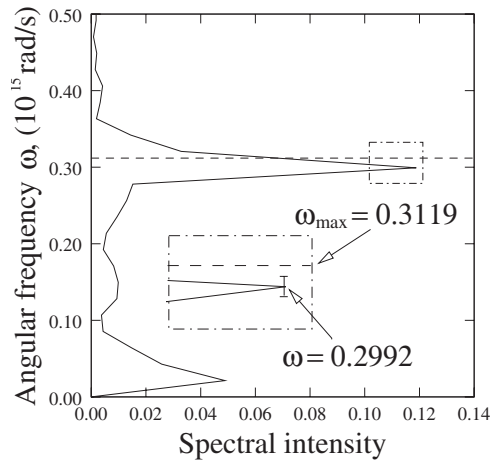

(c) $(5,5)$

FIG. 10. Power spectrum distribution in the $(7,7),(6,6)$, and $(5,5)$ armchair CNTs. 


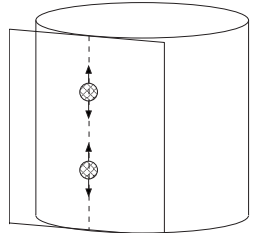

(a) Zigzag

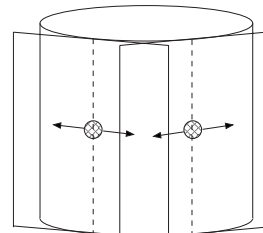

(b) Armchair
FIG. 11. Schematic illustration explaining the vibration mode in the zigzag and armchair CNTs.

$$
K_{l i \alpha}^{l^{\prime} j \beta}=\frac{\partial^{2} P}{\partial r_{l i \alpha} \partial r_{l^{\prime} j \beta}} .
$$

Figure 7(a) shows the frequency spectrum of the atom inside the circle $\eta$ in Fig. 6 during the high-energy state, where the dashed line in the figure denotes the upper bound of the phonon band $\omega_{\max }$. The spectrum is calculated by the discrete Fourier transform of the displacement-time relation of the atom during the high-energy state, where the sampling interval is $1 \mathrm{fs}$. The spectrum of the other atoms forming vibration modes (atoms inside circles in Fig. 6) is also analyzed, and the frequencies of their main peaks are within the error bar shown in Fig. 7(a). The dominant frequency exceeds $\omega_{\max }$ due to excitation of the localized vibration, which corresponds to a characteristic feature of the ILM. Furthermore, the vibration with a frequency above $\omega_{\max }$ continues for about 15 cycles (about 300 fs). These results imply excitation of the ILM. Figures 7(b) and 7(c) indicate that the ILM is also excited in the other zigzag CNTs, $(10,0)$ and $(8,0)$.

Figure 8 shows the change in the distribution of energy in the $(7,7) \mathrm{CNT}$. Although some atoms have high-energy momentarily, the state does not even continue for $50 \mathrm{fs}$, which is much shorter than that in the zigzag CNTs (300 fs). This implies that the ILM is not excited in the $(7,7)$ CNT. Figure 9 shows $\boldsymbol{V}_{i}$ in the $(7,7) \mathrm{CNT}$. Here, atoms vibrate in the circumferential direction. They do not form vibration modes with a fairly long lifetime. Figure 10(a) shows the frequency spectrum of the atom inside the circle $\xi$ in Fig. 9 during the high-energy state. The frequencies of the main peaks of the other atoms which have momentarily-large amplitude are within the error bar shown in Fig. 10(a). The dominant frequency is below $\omega_{\max }$, which indicates that the ILM is not excited in the $(7,7)$ CNT. Figures $10(\mathrm{~b})$ and $10(\mathrm{c})$ indicate that the ILM is not excited in the other armchair CNTs, $(6,6)$ and $(5,5)$, either.

In the zigzag CNTs, the optical vibration mode forms an in-plane vibration along the axial direction of the tube [Fig. 11(a)]. Since this possesses the strongest nonlinearity in the interaction between atoms, the ILM is excited in the zigzag CNTs. On the other hand, in the armchair CNTs, atoms vibrate in the circumferential direction [Fig. 11(b)]. As this out-of-plane vibration has weak nonlinearity, the ILM is not excited. Note, however, that a CNT with a large diameter has properties indistinguishable for a graphene sheet. Therefore, if the diameter of armchair CNTs is larger than a critical value, the ILM should also be able to exist in armchair CNTs. These points indicate that the ILM is dependent on the
TABLE II. Summary of simulation results.

\begin{tabular}{ccccc}
\hline $\begin{array}{c}\text { Chiral } \\
\text { index }\end{array}$ & $\begin{array}{c}\text { Vibration } \\
\text { direction }\end{array}$ & $\begin{array}{c}\text { Excitation } \\
\text { of ILM }\end{array}$ & $\begin{array}{c}\text { No. of } \\
\text { ILMs }\end{array}$ & $\begin{array}{c}\text { Average number } \\
\text { of ILM cycles }\end{array}$ \\
\hline$(12,0)$ & Axial & $\bigcirc$ & 5 & 14 \\
$(10,0)$ & Axial & $\bigcirc$ & 4 & 15 \\
$(8,0)$ & Axial & $\bigcirc$ & 4 & 15 \\
$(7,7)$ & Circumferential & $\times$ & & \\
$(6,6)$ & Circumferential & $\times$ & & \\
$(5,5)$ & Circumferential & $\times$ & & \\
\hline \hline
\end{tabular}

structure of the CNT. Further research is necessary to identify the critical bonding direction and diameter in the CNTs for the excitation of ILM. Table II summarizes the simulation results. In the zigzag CNTs, the number of ILMs in the system is estimated to be around five, and the ILMs continue for about 15 cycles, which is nearly equivalent to $300 \mathrm{fs}$. The reason why the number and the lifetime of the ILMs are almost the same among the zigzag CNTs is that the formation of vibration modes along the axial direction is independent of the diameter.

\section{CONCLUSION}

Molecular dynamics simulations were conducted in order to examine the excitation of the ILM in zigzag and armchair CNTs. Energy localization is observed in the $(12,0),(10,0)$, and $(8,0)$ zigzag CNTs, wherein two atoms vibrate. The optical modes, in which two neighboring atoms vibrate in the opposite direction along the axial direction of tube, are formed at the energy-localized areas. The frequency of the localized vibration in the zigzag CNTs exceeds the upper bound of the phonon band, which is a characteric feature of the ILM. These indicate excitation of the ILM.

Energy localization is not observed and the vibrations do not last a long time in the $(7,7),(6,6)$, and $(5,5)$ armchair CNTs, wherein atoms vibrate in the circumferential direction. The frequency in the armchair CNTs is below the upper bound of the phonon band.

Due to the in-plane vibration, the nonlinearity is the strongest in the optical mode of the zigzag CNTs. On the other hand, out-of-plane vibration of the optical mode in the armchair CNTs weakens the nonlinearity. Thus, the ILM is excited in the former while it is not in the latter.

In the future, we will analyze the vibrations of chiral CNTs in order to clarify the effect of structural differences on the excitation of the ILM in more detail. The excitation of the ILM in some chiral CNTs with almost the same diameter will be examined.

\section{ACKNOWLEDGMENTS}

This study was supported in part by the Center of Excellence for Research and Education on Complex Functional Mechanical Systems (COE program of the Ministry of Education, Culture, Sports, Science and Technology, Japan). 
${ }^{1}$ A. J. Sievers and S. Takeno, Phys. Rev. Lett. 61, 970 (1988).

${ }^{2}$ S. Takeno, K. Kisoda, and A. Sievers, Prog. Theor. Phys. Suppl. 94, 242 (1988).

${ }^{3}$ S. Flash and C. R. Willis, Phys. Rep. 295, 181 (1998).

${ }^{4}$ R. S. Mackay and S. Aubry, Nonlinearity 7, 1623 (1994).

${ }^{5}$ E. Trias, J. J. Mazo, and T. P. Orlando, Phys. Rev. Lett. 84, 741 (2000).

${ }^{6}$ P. Binder, D. Abraimov, A. V. Ustinov, S. Flach, and Y. Zolotaryuk, Phys. Rev. Lett. 84, 745 (2000).

${ }^{7}$ H. S. Eisenberg, Y. Silberberg, R. Morandotti, A. R. Boyd, and J. S. Aitchison, Phys. Rev. Lett. 81, 3383 (1998).

${ }^{8}$ M. Sato, B. E. Hubbard, A. J. Sievers, B. Ilic, D. A. Czaplewski, and H. G. Craighead, Phys. Rev. Lett. 90, 044102 (2003).

${ }^{9}$ M. Sato, B. E. Hubbard, A. J. Sievers, B. Ilic, and H. G. Craighead, Europhys. Lett. 66, 318 (2004).

${ }^{10}$ S. R. Bickham, S. A. Kiselev, and A. J. Sievers, Phys. Rev. B 47, 14206 (1993).

${ }^{11}$ S. A. Kiselev, S. R. Bickham, and A. J. Sievers, Phys. Rev. B 50, 9135 (1994).

${ }^{12}$ B. F. Feng, Y. Doi, and T. Kawahara, Physica D 214, 33 (2006).

${ }^{13}$ Y. Doi, Phys. Rev. E 68, 066608 (2003).
${ }^{14}$ J. Cuevas, C. Katerji, J. F. R. Archilla, J. C. Eilbeck, and F. M. Russell, Phys. Lett. A 315, 364 (2003).

${ }^{15}$ J. L. Marín, J. C. Eilbeck, and F. M. Russell, Phys. Lett. A 248, 225 (1998).

${ }^{16}$ F. M. Russell and J. C. Eilbeck, Europhys. Lett. 78, 10004 (2007).

${ }^{17}$ D. W. Brenner, Phys. Rev. B 42, 9458 (1990).

${ }^{18}$ Y. Yamayose, Y. Kinoshita, Y. Doi, A. Nakatani, and T. Kitamura, Europhys. Lett. 80, 40008 (2007).

${ }^{19}$ P. J. F. Harris, Carbon Nanotubes and Related Structures (Cambridge University Press, Cambridge, 1999).

${ }^{20}$ D. Tomanek and R. J. Enbody, Science and Application of Nanotubes (Kluwer, Dordrecht, 2000).

${ }^{21}$ M. S. Dresselhaus, G. Dresselhaus, and P. Avouris, Carbon Nanotubes (Springer, New York, 2001).

${ }^{22}$ A. V. Savin and Y. S. Kivshar, arXiv:0705.4482 (unpublished).

${ }^{23}$ J. Han, M. P. Anantram, R. L. Jaffe, J. Kong, and H. Dai, Phys. Rev. B 57, 14983 (1998).

${ }^{24}$ T. Cretegny, T. Dauxois, S. Ruffo, and A. Torcini, Physica D 121, 109 (1998).

${ }^{25}$ L. Verlet, Phys. Rev. 159, 98 (1967). 